\title{
Pseudorandom Generators from One-Way Functions: A Simple Construction for Any Hardness
}

\author{
Thomas Holenstein \\ ETH Zurich, Department of Computer Science, CH-8092, Zurich \\ thomahol@inf.ethz.ch
}

\begin{abstract}
In a seminal paper, Håstad, Impagliazzo, Levin, and Luby showed that pseudorandom generators exist if and only if one-way functions exist. The construction they propose to obtain a pseudorandom generator from an $n$-bit one-way function uses $\mathcal{O}\left(n^{8}\right)$ random bits in the input (which is the most important complexity measure of such a construction). In this work we study how much this can be reduced if the one-way function satisfies a stronger security requirement. For example, we show how to obtain a pseudorandom generator which satisfies a standard notion of security using only $\mathcal{O}\left(n^{4} \log ^{2}(n)\right)$ bits of randomness if a one-way function with exponential security is given, i.e., a one-way function for which no polynomial time algorithm has probability higher than $2^{-c n}$ in inverting for some constant $c$.

Using the uniform variant of Impagliazzo's hard-core lemma given in [7] our constructions and proofs are self-contained within this paper, and as a special case of our main theorem, we give the first explicit description of the most efficient construction from [6].
\end{abstract}

\section{Introduction}

A pseudorandom generator is a deterministic function which takes a uniform random bit string as input and outputs a longer bit string which cannot be distinguished from a uniform random string by any polynomial time algorithm. This concept, introduced in the fundamental papers of Yao [16] and Blum and Micali [1] has many uses. For example, it immediately gives a semantically secure cryptosystem: the input of the pseudorandom generator is the key of the cryptosystem, and the output is used as a one-time pad. Other uses of pseudorandom generators include the construction of pseudorandom functions [2], pseudorandom permutations [11], statistically binding bit commitment [13], and many more.

Such a pseudorandom generator can be obtained from an arbitrary one-way function, as shown in [6]. The given construction is not efficient enough to be used in practice, as it requires $\mathcal{O}\left(n^{8}\right)$ bits of input randomness (for example, if one would like to have approximately the security of a one-way function with $n=100$ input bits, the resulting pseudorandom generator would need several petabits of input, which is clearly impractical). On the other hand, it is possible 
to obtain a pseudorandom generator very efficiently from an arbitrary one-way permutation [4] or from an arbitrary regular one-way function [3] (see also [5]), i.e., a one-way function where every image has the same number of preimages. In other words, if we have certain guarantees on the combinatorial structure of the one-way function, we can get very efficient reductions.

In this paper we study the question whether a pseudorandom generator can be obtained more efficiently under a stronger assumption on the computational difficulty of the one-way function. In particular, assume that the one-way function is harder to invert than usually assumed. In this case, one single invocation of the one-way function could be more useful, and fewer invocations might be needed. We will see that is indeed the case, even if the pseudorandom generator is supposed to inherit a stronger security requirement from the one-way function, and not only if it is supposed to satisfy the standard security notion.

\section{Overview of the Construction}

The construction given in [6] uses several stages: first the one-way function is used to construct a false entropy generator, i.e., a function whose output is computationally indistinguishable from a distribution with more entropy. (This is the technically most difficult part of the construction and the security proof can be significantly simplified by using the uniform hard-core lemma from [7].) Next, the false entropy generator is used to construct a pseudoentropy generator (a function whose output is computationally indistinguishable from a distribution which has more entropy than the input), and finally a pseudorandom generator is built on top of that. If done in this way, their construction is very inefficient (requiring inputs of length $\mathcal{O}\left(n^{34}\right)$ ), but it is also sketched in [6] how to "unroll" the construction in order to obtain an $\mathcal{O}\left(n^{10}\right)$ construction. Similarly it is mentioned that an $\mathcal{O}\left(n^{8}\right)$ construction is possible (by being more careful).

In this work we explicitly describe an $\mathcal{O}\left(n^{8}\right)$ construction (in an unrolled version the construction we describe is the one sketched in [6]). Compared to [6] we choose a different way of presenting this construction; namely we use a twostep approach (see Figure 1). First, (in Section 4) we use the one-way function to construct a pair $(g, P)$ where $g$ is an efficiently evaluable function and $P$ is a predicate. The pair will satisfy that predicting $P(x)$ from $g(x)$ is computationally

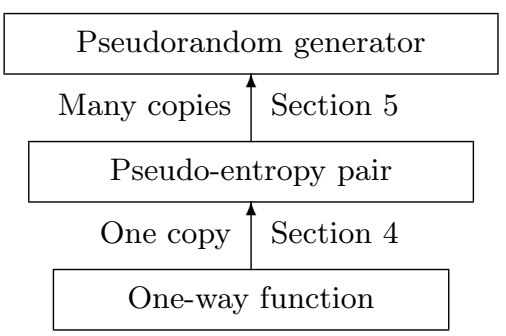

Fig. 1. Overview of our construction 
difficult (in particular, more difficult than it would be information theoretically). In [5] the term pseudo-entropy pair is coined for such a pair and we will use this term as well. In a second step we use many instances of such a pseudo-entropy pair to construct a pseudorandom generator.

Further, we generalize the construction to the case where stronger security guarantees on the one-way function are given. This enables us to give more efficient reductions under stronger assumptions.

Indepenently of this work, Haitner, Harnik, and Reingold [5] give a better method to construct a pseudo-entropy pair from a one-way function. Their construction has the advantage that the entropy of $P(x)$ given $g(x)$ can be estimated, which makes the construction of the pseudorandom generator from the pseudo-entropy pair more efficient.

\section{Definitions and Result}

\subsection{Definitions and Notation}

Definition 1. A one-way function with security $s(n)$ against $t(n)$-bounded inverters is an efficiently evaluable family of functions $f:\{0,1\}^{n} \rightarrow\{0,1\}^{m}$ such that for any algorithm running in time at most $t(n)$

$$
\operatorname{Pr}_{x \leftarrow R_{R}\{0,1\}^{n}}[f(A(f(x)))=f(x)]<\frac{1}{s(n)}
$$

for all but finitely many $n$.

For example the standard notion of a one-way function is a function which is oneway with security $p(n)$ against $p(n)$-bounded inverters for all polynomials $p(n)$.

In [15] it is shown that a random permutation is $2^{n / 10}$-secure against $2^{n / 5}$ bounded inverters, and also other reasons are given why it is not completely unreasonable to assume the existence of one-way permutations with exponential security. In our main theorem we can use one-way functions with exponential security, a weaker primitive than such permutations.

Definition 2. A pseudorandom-generator with security $s(\ell)$ against $t(\ell)$-bounded distinguishers is an efficiently evaluable family of (expanding) functions $h:\{0,1\}^{\ell} \rightarrow\{0,1\}^{\ell+1}$ such that for any algorithm running in time at most $t(\ell)$

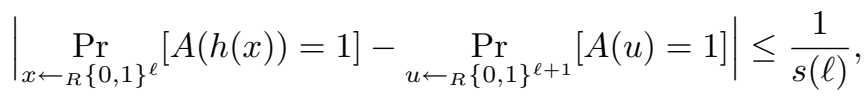

for all but finitely many $\ell$.

The standard notion of a pseudorandom generator is a pseudorandom generator with security $p(\ell)$ against $p(\ell)$-bounded distinguishers, for all polynomials $p(\ell)$.

As mentioned above, we use pseudo-entropy pairs as a step in our construction. For such a pair of functions we first define the advantage an algorithm $A$ has in predicting $P(w)$ from $g(w)$ (by convention, we use the letter $w$ to denote the input here). 
Definition 3. For any algorithm A, any function $g:\{0,1\}^{n} \rightarrow\{0,1\}^{m}$ and any predicate $P:\{0,1\}^{n} \rightarrow\{0,1\}$, the advantage of $A$ in predicting $P$ given $g$ is

$$
\operatorname{Adv}^{A}(g, P):=2\left(\operatorname{Pr}_{w \leftarrow R\{0,1\}^{n}}[A(g(w))=P(w)]-\frac{1}{2}\right) .
$$

The following definition of a pseudo-entropy pair contains (somewhat surprisingly) the conditioned entropy $H(P(W) \mid g(W))$; we give an explanation below.

Definition 4. A pseudo-entropy pair with gap $\phi(n)$ against $t(n)$-bounded predictors is a pair $(g, P)$ of efficiently evaluable functions $g:\{0,1\}^{n} \rightarrow\{0,1\}^{m}$ and $P:\{0,1\}^{n} \rightarrow\{0,1\}$ such that for any algorithm $A$ running in time $t(n)$

$$
\operatorname{Adv}^{A}(g, P) \leq 1-H(P(W) \mid g(W))-\phi,
$$

for all but finitely many $n$ (where $W$ is uniformly distributed over $\{0,1\}^{n}$ ).

The reader might think that it would be more natural if we used the best advantage for computationally unbounded algorithms (i.e., the information theoretic advantage), instead of $1-H(P(W) \mid g(W))$. Then $\phi$ would be the gap which comes from the use of $t(n)$-bounded predictors. We quickly explain why we chose the above definition. First, to get an intuition for the expression $1-H(P(W) \mid g(W))$, assume that the pair $(g, P)$ has the additional property that for every input $w$, $g(w)$ either fixes $P(w)$ completely or does not give any information about it, i.e., for a fixed value $v$ either $H(P(W) \mid g(W)=v)=1$ or $H(P(W) \mid g(W)=v)=0$ holds. Then, a simple computation shows that $1-H(P(W) \mid g(W))$ is a tight upper bound on the advantage of computationally unbounded algorithms, i.e., in this case our definition coincides with the above "more natural definition". We mention here that the pairs $(g, P)$ we construct will be close to pairs which have this property. If there are values $v$ such that $0<H(P(W) \mid g(W)=v)<1$, the expression $1-H(P(W) \mid g(W))$ is not an upper bound anymore and in fact one might achieve significantly greater advantage than $1-H(P(W) \mid g(W))$. Therefore in this case, Definition 4 requires something stronger than the "more natural definition", and, consequently, constructing a pseudorandom generator from a pseudo-entropy pair becomes easier. ${ }^{1}$

We use $\|$ to denote concatenation of strings, ax denotes the multiplication of bitstrings $a$ and $x$ over $\mathrm{GF}\left(2^{n}\right)$ (with an arbitrary representation), and $\left.x\right|_{\lambda}$ denotes the first $\lfloor\lambda\rfloor$ bits of the bit string $x$. For fixed $x$ and $\bar{x}, x \neq \bar{x}$, the probability that $\left.(a x)\right|_{i}$ equals $\left.(a \bar{x})\right|_{i}$ for uniformly chosen $a$ can be computed as

$$
\operatorname{Pr}_{a \leftarrow\{0,1\}^{n}}\left[\left.(a x)\right|_{i}=\left.(a \bar{x})\right|_{i}\right]=\operatorname{Pr}_{a \leftarrow\{0,1\}^{n}}\left[\left.(a(x-\bar{x}))\right|_{i}=0^{i}\right]=2^{-i}
$$

an expression we will use later.

${ }^{1}$ In fact, we do not know a direct way to construct a pseudorandom generator from a pseudo-entropy pair with the "more natural definition". 
For bitstrings $x$ and $r$ of the same length $n$ we use $x \odot r:=\bigoplus_{i=1}^{n} x_{i} r_{i}$ for the inner product. We use the convention that $f^{-1}(y):=\left\{x \in\{0,1\}^{n} \mid f(x)=y\right\}$, i.e., $f^{-1}$ returns a set.

For two distributions $P_{X_{0}}$ and $P_{X_{1}}$ over $\mathcal{X}$ the statistical distance is

$$
\Delta\left(X_{0}, X_{1}\right):=\frac{1}{2} \sum_{x \in \mathcal{X}}\left|P_{X_{0}}(x)-P_{X_{1}}(x)\right| .
$$

We also say that a distribution is $\varepsilon$-close to another distribution if the statistical distance of the distributions is at most $\varepsilon$. For a distribution $P_{X}$ over $\mathcal{X}$ the min-entropy is $H_{\infty}(X):=-\log \left(\max _{x \in \mathcal{X}} P_{X}(x)\right)$. For joint distributions $P_{X Y}$ over $\mathcal{X} \times \mathcal{Y}$ the conditional min-entropy is defined with $H_{\infty}(X \mid Y):=$ $\min _{y \in \mathcal{Y}} H_{\infty}(X \mid Y=y)$.

Finally, we define $[n]:=\{1, \ldots, n\}$.

\subsection{Result}

We give a general construction of a pseudorandom generator from a one-way function. The construction is parametrized by two parameters $\varepsilon$ and $\phi$. The parameter $\varepsilon$ should be chosen such that it is smaller than the target indistinguishability of the pseudorandom generator: an algorithm which distinguishes the output of the pseudorandom generator with advantage less than $\varepsilon$ will not help us in inverting $f$. The second parameter $\phi$ should be chosen such that the given one-way function cannot be inverted with probability more than about $2^{-n \phi}$ (as an example, for standard security notions choosing $\phi=\frac{1}{n}$ and $\varepsilon=2^{-n}$ would be reasonable - these should be considered the canonical choices).

Theorem 1. Let functions $f:\{0,1\}^{n} \rightarrow\{0,1\}^{m}, \phi: \mathbb{N} \rightarrow[0,1], \varepsilon: \mathbb{N} \rightarrow[0,1]$ be given, computable in time poly $(n)$, and satisfying $2^{-n} \leq \varepsilon \leq \frac{1}{n} \leq \phi$.

There exists an efficient to evaluate oracle function $h_{\varepsilon, \phi}^{f}$ with the following properties:

$-h_{\varepsilon, \phi}^{f}$ is expanding,

- $h_{\varepsilon, \phi}^{f}$ has input of length $\mathcal{O}\left(\frac{n^{4}}{\phi^{4}} \log \left(\frac{1}{\varepsilon}\right)\right)$, and

- an algorithm $A$ which distinguishes the output of $h_{\varepsilon, \phi}^{f}$ from a uniform bit string with advantage $\gamma$ can be used to get an oracle algorithm which inverts $f$ with probability $\mathcal{O}\left(\frac{1}{n^{3}}\right) 2^{-n \phi}$, using poly $\left(n, \frac{1}{\gamma-\varepsilon}\right)$ calls to $A$.

For example, if we set $\phi:=\log (n) / n$ and $\varepsilon:=n^{-\log (n)}=2^{-\log ^{2}(n)}$ and use a standard one-way function in the place of $f$, then $h_{\varepsilon, \phi}^{f}$ will be a standard pseudorandom generator, using $\mathcal{O}\left(n^{8}\right) \operatorname{bits}^{2}$ of randomness.

Corollary 1. Assume that $f:\{0,1\}^{n} \rightarrow\{0,1\}^{m}$ is a one-way function with security $p(n)$ against $p(n)$-bounded inverters, for all polynomials $p(n)$. Then there exists a pseudorandom generator $h:\{0,1\}^{\ell} \rightarrow\{0,1\}^{\ell+1}$ with security $p(\ell)$ against $p(\ell)$-bounded distinguishers, for all polynomials $p(\ell)$. The construction calls the one-way function for one fixed $n$ dependent of $\ell$ and satisfies $\ell \in \mathcal{O}\left(n^{8}\right)$.

$\overline{2}$ This could be insignificantly reduced by choosing $\varepsilon$ slightly bigger. 
Alternatively if we have a much stronger one-way function which no polynomial time algorithm can invert with better probability than $2^{-c n}$ for some constant $c$, we can set $\phi$ to some appropriate small constant and $\varepsilon:=n^{-\log (n)}$, which gives us a pseudorandom generator using $\mathcal{O}\left(n^{4} \log ^{2}(n)\right)$ bits of input:

Corollary 2. Assume that $f:\{0,1\}^{n} \rightarrow\{0,1\}^{m}$ is a one-way function with security $2^{-c n}$ against $p(n)$-bounded inverters, for some constant $c$ and all polynomials $p(n)$. Then there exists a pseudorandom generator $h:\{0,1\}^{\ell} \rightarrow\{0,1\}^{\ell+1}$ with security $p(\ell)$ against $p(\ell)$-bounded distinguishers, for all polynomials $p(\ell)$. The construction calls the one-way function for one fixed $n$ dependent of $\ell$, and satisfies $\ell \in \mathcal{O}\left(n^{4} \log ^{2}(n)\right)$.

If we want a pseudorandom generator with stronger security we set $\varepsilon$ smaller in our construction. For example, if a one-way function $f$ has security $2^{\text {cn }}$ against $2^{\text {cn }}$ bounded distinguishers, we set $\phi$ (again) to an appropriate constant and $\varepsilon:=2^{-n}$. With these parameters our construction needs $\mathcal{O}\left(n^{5}\right)$ input bits, and, for an appropriate constant $d$, an algorithm with distinguishing advantage $2^{-d n}$, and running in time $2^{d n}$, can be used to get an inverting algorithm which contradicts the assumption about $f$. (A corollary similar to the ones before could be formulated here).

The proof of Theorem 1 is in two steps (see Figure 1). In Section 4 we use the Goldreich-Levin Theorem and two-universal hash-functions to obtain a pseudoentropy pair. In Section 5 we show how such a pair can be used to obtain a pseudorandom generator.

\subsection{Extractors}

Informally, an extractor is a function which can extract a uniform bit string from a random string with sufficient min-entropy. The following well known left-over hash lemma from [10] shows that multiplication over $\operatorname{GF}\left(2^{n}\right)$ with a randomly chosen string $a$ and then cutting off an appropriate number of bits can be used to extract randomness. For completeness we give a proof (adapted from [12]).

Lemma 1 (Left-over hash lemma). Let $x \in\{0,1\}^{n}$ be chosen according to any source with min-entropy $\lambda$. Then, for any $\varepsilon>0$, and uniform random $a$, the distribution of $\left(\left.(a x)\right|_{\lambda-2 \log \left(\frac{1}{\varepsilon}\right)} \| a\right)$ is $\frac{\varepsilon}{2}$-close to a uniform bit string of length $\left\lfloor\lambda-2 \log \left(\frac{1}{\varepsilon}\right)\right\rfloor+n$.

Proof. Let $m:=\left\lfloor\lambda-2 \log \left(\frac{1}{\varepsilon}\right)\right\rfloor$, and $P_{V A}$ be the distribution of $\left.(a x)\right|_{m} \| a$. Further, let $P_{U}$ be the uniform distribution over $\{0,1\}^{m+n}$. Using the Cauchy-Schwartz inequality $\left(\sum_{i=1}^{k} a_{i}\right)^{2} \leq k \sum_{i=1}^{k} a_{i}^{2}$ we obtain for the statistical distance in question

$$
\Delta(V A, U)=\frac{1}{2} \sum_{v \in\{0,1\}^{m}, a \in\{0,1\}^{n}}\left|P_{V A}(v, a)-\frac{1}{2^{n} 2^{m}}\right|
$$




$$
\begin{aligned}
& \leq \frac{1}{2} \sqrt{2^{n} 2^{m}} \sqrt{\sum_{v, a} P_{V A}^{2}(v, a)-2 \sum_{v, a} \frac{P_{V A}(v, a)}{2^{n} 2^{m}}+\sum_{v, a}\left(\frac{1}{2^{n} 2^{m}}\right)^{2}} \\
& =\frac{1}{2} \sqrt{2^{n} 2^{m}} \sqrt{\sum_{v, a} P_{V A}^{2}(v, a)-\frac{1}{2^{n} 2^{m}}} .
\end{aligned}
$$

Let now $X_{0}$ and $X_{1}$ be independently distributed according to $P_{X}$ (i.e., the source with min-entropy $\lambda$ ). Further, let $A_{0}$ and $A_{1}$ be independent over $\{0,1\}^{n}$. The collision probability of the output distribution is

$$
\operatorname{Pr}\left[\left(\left.\left(X_{0} A_{0}\right)\right|_{m} \| A_{0}\right)=\left(\left.\left(X_{1} A_{1}\right)\right|_{m} \| A_{1}\right)\right]=\sum_{v, a} P_{V A}^{2}(v, a) .
$$

Thus we see that equation (2) gives an un upper bound on $\Delta(V A, U)$ in terms of the collision probability of two independent invocations of the hash-function on two independent samples from the distribution $P_{X}$. We can estimate this collision probability as follows:

$$
\begin{aligned}
\operatorname{Pr}[ & \left.\left(\left.\left(X_{0} A_{0}\right)\right|_{m} \| A_{0}\right)=\left(\left.\left(X_{1} A_{1}\right)\right|_{m} \| A_{1}\right)\right] \\
& =\operatorname{Pr}\left[A_{0}=A_{1}\right] \operatorname{Pr}\left[\left.\left(X_{0} A_{0}\right)\right|_{m}=\left.\left(X_{1} A_{0}\right)\right|_{m}\right] \\
& \leq \operatorname{Pr}\left[A_{0}=A_{1}\right]\left(\operatorname{Pr}\left[X_{0}=X_{1}\right]+\operatorname{Pr}\left[\left.\left(X_{0} A_{0}\right)\right|_{m}=\left.\left(X_{1} A_{0}\right)\right|_{m} \mid X_{0} \neq X_{1}\right]\right) \\
& \leq \frac{1}{2^{n}}\left(\frac{1}{2^{m+2} \log (1 / \varepsilon)}+\frac{1}{2^{m}}\right)=\frac{1+\varepsilon^{2}}{2^{n} 2^{m}},
\end{aligned}
$$

where we used (1) in the last inequality. We now insert (3) into (2) and get $\Delta(V A, U) \leq \frac{\varepsilon}{2}$.

Using the usual definition of an extractor, the above lemma states that multiplying with a random element of $\operatorname{GF}\left(2^{n}\right)$ and then cutting off the last bits is a strong extractor. Consequently, we will sometimes use the notation $\operatorname{Ext}_{m}(x, a)$ to denote the function $\operatorname{Ext}_{m}(x, a):=\left.(a x)\right|_{m} \| a$, extracting $\lfloor m\rfloor$ bits from $x$.

Further we use the following proposition on independent repetitions from [8], which is a quantitative version of the statement that for $k$ independent repetitions of random variables, the min-entropy of the resulting concatenation is roughly $k$ times the (Shannon-)entropy of a single instance (assuming $k$ large enough and tolerating a small probability that something improbable occured). A similar lemma with slightly weaker parameters is given in [10] (the latter would be sufficient for our application, but the expression from [8] is easier to use).

Proposition 1. Let $\left(X_{1}, Y_{1}\right), \ldots,\left(X_{k}, Y_{k}\right)$ i.i.d. according to $P_{X Y}$. For any $\varepsilon$ there exists a distribution $P_{\bar{X}} \bar{Y}$ which has statistical distance at most $\frac{\varepsilon}{2}$ from $\left(X_{1}, \ldots, X_{k}, Y_{1}, \ldots, Y_{k}\right)$ and satisfies

$$
H_{\infty}(\bar{X} \mid \bar{Y}) \geq k H(X \mid Y)-6 \sqrt{k \log (1 / \varepsilon)} \log (|\mathcal{X}|) .
$$

We can combine the above propositions as follows: 
Lemma 2. Let $k$, $\varepsilon$ with $k>\log (1 / \varepsilon)$ be given. Let $\left(X_{1}, Y_{1}\right), \ldots,\left(X_{k}, Y_{k}\right)$ i.i.d. according to $P_{X Y}$ over $\mathcal{X} \times \mathcal{Y}$ with $\mathcal{X} \subseteq\{0,1\}^{n}$. Let $A$ be uniform over $\{0,1\}^{k n}$.

Then,

$$
\operatorname{Ext}_{k H(X \mid Y)-8 \log (|\mathcal{X}|) \sqrt{k \log (1 / \varepsilon)}}\left(X_{1}\|\cdots\| X_{k}, A\right)\left\|Y_{1}\right\| \cdots \| Y_{k}
$$

is $\varepsilon$-close to $U \times Y^{k}$, where $U$ is an independent uniform chosen bitstring of length $\lfloor k H(X \mid Y)-8 \log (|\mathcal{X}|) \sqrt{k \log (1 / \varepsilon)}\rfloor+k n$.

Proof. Combine Lemma 1 and Proposition 1.

\section{A Pseudo-Entropy Pair from Any One-Way Function}

The basic building block we use to get a pseudo-entropy pair is the following theorem by Goldreich and Levin [4] (recall that $x \odot r=x_{1} r_{1} \oplus \cdots \oplus x_{n} r_{n}$ is the inner product of $x$ and $r$ ):

Proposition 2 (Goldreich-Levin). There is an oracle algorithm $B^{(\cdot)}$ such that for any $x \in\{0,1\}^{n}$ and any oracle $A$ satisfying

$$
\operatorname{Pr}_{r \leftarrow R}\{0,1\}^{n}[A(r)=x \odot r] \geq \frac{1}{2}+\gamma
$$

$B^{A}$ does $\mathcal{O}\left(\frac{n}{\gamma^{2}}\right)$ queries to $A$ and then efficiently outputs a list of $\mathcal{O}\left(\frac{1}{\gamma^{2}}\right)$ elements such that $x$ is in the list with probability $\frac{1}{2}$.

This proposition implies that for any one-way function $f$, no efficient algorithm will be able to predict $x \odot r$ from $f(x)$ and $r$ much better than random guessing, as otherwise the one-way function can be broken.

This suggests the following method to get a pseudo-entropy pair: if we define $g(x, r):=f(x) \| r$ and $P(x, r):=x \odot r$, then predicting $P(x, r)$ from $g(x, r)$ is computationally hard. The problem with this approach is that since $f(x)$ may have many different preimages, $H(P(X, R) \mid g(X, R)) \approx 1$ is possible. In this case, $P(x, r)$ would not only be computationally unpredictable, but also information theoretically unpredictable, and thus $(g, P)$ will not be a pseudo-entropy pair.

The solution of this problem (as given in [6]), is that one additionally extracts some information of the input $x$ to $f$; the amount of information extracted is also random. The idea is that in case one is lucky and extracts roughly $\log \left(\left|f^{-1}(f(x))\right|\right)$ bits, then these extracted bits and $f(x)$ fix $x$ in an information theoretic way, but computationally $x \odot r$ is still hard to predict because of Proposition 2.

Thus, we define functions $g:\{0,1\}^{4 n} \rightarrow\{0,1\}^{m+4 n}$ and $P:\{0,1\}^{4 n} \rightarrow\{0,1\}$ as follows (where $i \in[n]$ is a number ${ }^{3}, x, a$, and $r$ are bitstrings, and we ignore padding which should be used to get $\left.(a x)\right|_{i}$ to length $n$ )

${ }^{3}$ Technically, we should choose $i$ as a uniform number from $[n]$. We can use an $n$ bit string to choose a uniform number from $\left[2^{n}\right]$ and from this we can get an "almost" uniform number from $[n]$ (for example by computing the remainder when dividing by $n$ ). This only gives an exponentially small error which we ignore from now on. 


$$
\begin{aligned}
g(x, i, a, r) & :=f(x)\|i\| a\left\|\left.(a x)\right|_{i}\right\| r \\
P(x, i, a, r) & :=x \odot r .
\end{aligned}
$$

We will alternatively write $g(w)$ and $P(w)$, i.e., we use $w$ as an abbreviation for $(x, i, a, r)$. We will prove that $(g, P)$ is a pseudo-entropy pair in case $f$ is a one-way function. Thus we show that no algorithm exceeds advantage $1-$ $H(P(W) \mid g(W))-\phi$ in predicting $P(w)$ from $g(w)$ (the gap $\phi$ does not appear in the construction, but the pair will have a bigger gap if the one-way function satisfies as stronger security requirement, as we will see).

We first give an estimate on $H(P(W) \mid g(W))$. The idea is that we can distinguish two cases: either $i \geq \log \left(\left|f^{-1}(f(x))\right|\right)$, in which case $H(P(W) \mid g(W)) \approx 0$, since $\left.(a x)\right|_{i}$, a, and $f(x)$ roughly fix $x$, or $i<\log \left(\left|f^{-1}(f(x))\right|\right)$, in which case $H(P(W) \mid g(W)) \approx 1$.

Lemma 3. For the functions $g$ and $P$ as defined above

$$
H(P(W) \mid g(W)) \leq \frac{\mathrm{E}_{x \leftarrow R}\{0,1\}^{n}\left[\log \left(\left|f^{-1}(f(x))\right|\right)\right]+2}{n}
$$

Proof. From (1) and the union bound we see that if $i>\log \left(\left|f^{-1}(y)\right|\right)$ the probability that $x$ is not determined by the output of $g$ is at most $2^{-\left(i-\log \left(\left|f^{-1}(y)\right|\right)\right)}$. This implies $H(P(W) \mid g(W), f(X)=y, I=i) \leq 2^{-\left(i-\log \left(\left|f^{-1}(y)\right|\right)\right)}$, and thus

$$
\begin{aligned}
H(P(W) \mid g(W)) & =\frac{1}{2^{n}} \sum_{x \in\{0,1\}^{n}} H(P(W) \mid g(W), f(X)=f(x)) \\
& =\frac{1}{2^{n}} \sum_{x \in\{0,1\}^{n}} \frac{1}{n} \sum_{i=1}^{n} H(P(W) \mid g(W), f(X)=f(x), I=i) \\
& \leq \frac{1}{2^{n}} \sum_{x \in\{0,1\}^{n}}\left(\frac{\log \left(\left|f^{-1}(f(x))\right|\right)}{n}\right. \\
& \leq \frac{1}{2^{n}} \sum_{x \in\{0,1\}^{n}} \frac{\log \left(\left|f^{-1}(f(x))\right|\right)+2}{n} 2^{\left.\left.-\left(i-\log \left(\left|f^{-1}(f(x))\right|\right)\right)\right)\right)} \\
& =\frac{\mathrm{E}_{x \leftarrow R\{0,1\}^{n}}\left[\log \left(\left|f^{-1}(f(x))\right|\right)\right]+2}{n} .
\end{aligned}
$$

We can now show that $(g, P)$ is a pseudo-entropy pair. For this, we show that any algorithm which predicts $P$ from $g$ with sufficient probability can be used to invert $f$. Recall that $\phi$ is usually $\frac{1}{n}$.

Lemma 4. Let $f:\{0,1\}^{n} \rightarrow\{0,1\}^{m}$ and $\phi: \mathbb{N} \rightarrow[0,1]$ be computable in time $\operatorname{poly}(n)$. Let functions $g$ and $P$ be as defined above. There exists an oracle 
algorithm $B^{(\cdot)}$ such that, for any $A$ which has advantage $\operatorname{Adv}^{A}\left(g^{f}, P^{f}\right) \geq 1-$ $H\left(P^{f}(W) \mid g^{f}(W)\right)-\phi$ in predicting $P^{f}$ from $g^{f}, B^{A}$ inverts $f$ with probability $\Omega\left(\frac{1}{n^{3}}\right) 2^{-n \phi}$ and $\mathcal{O}\left(n^{3}\right)$ calls to $A$.

We find it convenient to present our proof using random experiments called "games", similar to the method presented in [14].

Proof. Assume that a given algorithm $A(y, i, a, z, r)$ has an advantage exceeding the bound in the lemma in predicting $P$ from $g$. To invert a given input $y=f(x)$, we will choose $i, a$, and $z$ uniformly at random. Then we run the GoldreichLevin algorithm using $A(y, i, a, z, \cdot)$, i.e., the Goldreich-Levin calls $A(y, i, a, z, r)$ for many different $r$, but always using the same $y, i, a$, and $z$. This gives us a list $L$ containing elements from $\{0,1\}^{n}$. For every $\bar{x} \in L$ we check whether $f(\bar{x})=y$. If at least one $\bar{x} \in L$ satisfies this we succeeded in inverting $f$.

In order to see whether this approach is successful, we first define $\alpha$ to be the advantage of $A$ for a fixed $y, i, a$ and $z$ in predicting $\bar{x} \odot r$ for a preimage $\bar{x}$ of $y$ :

$$
\alpha(y, i, a, z):=\max _{\bar{x} \in f^{-1}(y)}\left(2 \operatorname{Pr}_{r \leftarrow\{0,1\}^{n}}[A(y, i, a, z, r)=\bar{x} \odot r]-1\right) .
$$

We maximize over all possible $\bar{x} \in f^{-1}(y)$, since it is sufficient if the above method finds any preimage of $y$. We will set the parameters of the algorithm such that it succeeds with probability $\frac{1}{2}$ if $\alpha(y, i, a, z)>\frac{1}{4 n}$ (i.e., with probability $\frac{1}{2}$ the list returned by the algorithm contains $\bar{x})$. It is thus sufficient to show for uniformly chosen $x, i, a$, and $z$ the inequality $\alpha(f(x), i, a, z)>\frac{1}{4 n}$ is satisfied with probability $\Omega\left(\frac{1}{n^{3}}\right) 2^{-n \phi}$.

Together with Lemma 3, the requirement of this lemma implies that in the following Game 0 the expectation of the output is at least $1-H\left(P^{f}(W) \mid g^{f}(W)\right)-$ $\phi \geq 1-\frac{1}{n} \mathrm{E}_{x}\left[\log \left(\left|f^{-1}(f(x))\right|\right)\right]-\frac{2}{n}-\phi$ (this holds even without the maximization in the definition of $\alpha$ and using $\bar{x}=x$ instead - clearly, the maximization cannot reduce the expected output of Game 0).

\section{Game 0:}

$$
\begin{aligned}
& x \leftarrow_{R}\{0,1\}^{n}, y:=f(x), i \leftarrow_{R}[n] \\
& a \leftarrow_{R}\{0,1\}^{n}, z:=\left.(a x)\right|_{i} \\
& \text { output } \alpha(y, i, a, z)
\end{aligned}
$$

Note that even though we can approximate $\alpha(y, i, a, z)$ we do not know how to compute the exact value in reasonable time. However, we do not worry about finding an efficient implementation of our games.

If $i$ is much larger than $\log \left(\left|f^{-1}(y)\right|\right)$ then predicting $P(w)$ from $g(w)$ is not very useful in order to invert $f$, since $\left.(a x)\right|_{i}$ gives much information about $x$ which we do not have if we try to invert $y$. Thus, we ignore the cases where $i$ is much larger than $\log \left(\left|f^{-1}(y)\right|\right)$ in Game 1 .

\section{Game 1:}

$$
\begin{aligned}
& x \leftarrow_{R}\{0,1\}^{n}, y:=f(x), i \leftarrow_{R}[n] \\
& \text { if } i \leq \log \left(\left|f^{-1}(y)\right|\right)+n \phi+3 \text { then } \\
& a \leftarrow_{R}\{0,1\}^{n}, z:=\left.(a x)\right|_{i}
\end{aligned}
$$




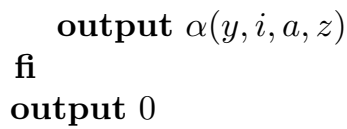

It is not so hard to see that the probability that the if clause fails is at most $1-\frac{1}{n} E_{x}\left[\log \left(\left|f^{-1}(f(x))\right|\right)\right]-\frac{3}{n}-\phi$. Thus, in Game 1 the expectation of the output is at least $\frac{1}{n}$ (because the output only decreases in case the if clause fails, and in this case by at most one).

In Game 2, we only choose the first $j$ bits of $z$ as above, where $j$ is chosen such that these bits will be $\frac{1}{4 n}$-close to uniform (this will be used later). We fill up the rest of $z$ with the best possible choice; clearly, this cannot decrease the expectation of the output.

\section{Game 2:}

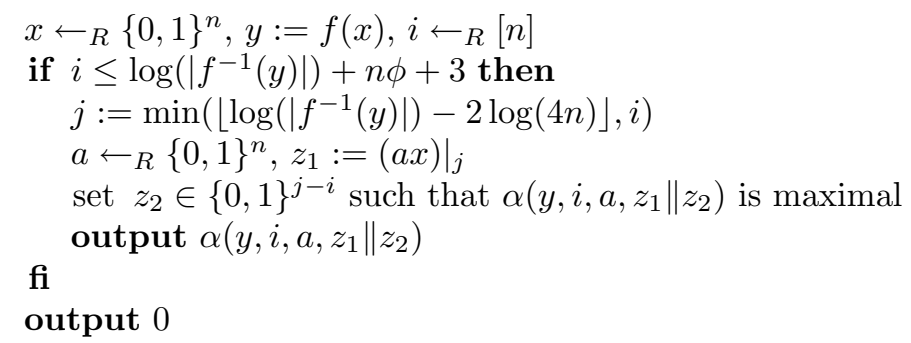

We now chose $z_{1}$ uniformly at random. Lemma 1 implies that the statistical distance of the previous distribution of $z_{1}$ to the uniform distribution (given $a, i$, and $y$ but not $x$ ) is at most $\frac{1}{4 n}$. Thus, the expecation of the output is at least $\frac{1}{2 n}$.

\section{Game 3:}

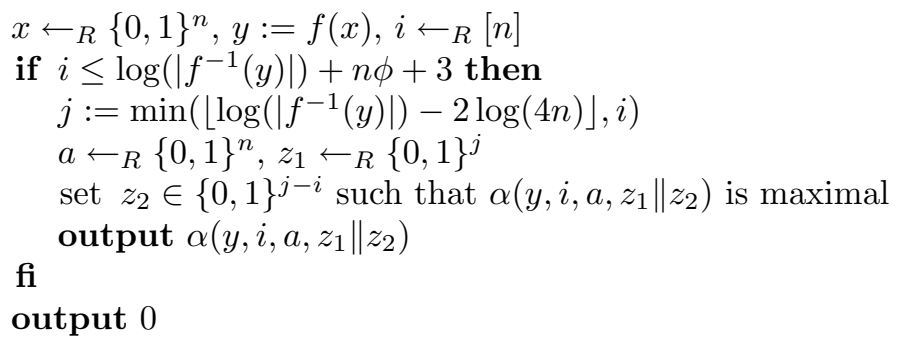

As mentioned above, we will be satisfied if we have values $y, i, a,\left(z_{1} \| z_{2}\right)$ such that $\alpha\left(y, i, a, z_{1} \| z_{2}\right) \geq \frac{1}{4 n}$. In Game 4 , we thus do not compute the expectation of $\alpha$ anymore, but only output success if this is satisfied, and fail otherwise.

Game 4:

$$
\begin{aligned}
& x \leftarrow_{R}\{0,1\}^{n}, y:=f(x), i \leftarrow_{R}[n] \\
& \text { if } i \leq \log \left(\left|f^{-1}(y)\right|\right)+n \phi+3 \text { then } \\
& \quad j:=\min \left(\left\lfloor\log \left(\left|f^{-1}(y)\right|\right)-2 \log (4 n)\right\rfloor, i\right) \\
& a \leftarrow_{R}\{0,1\}^{n}, z_{1} \leftarrow_{R}\{0,1\}^{j} \\
& \text { set } z_{2} \in\{0,1\}^{j-i} \text { such that } \alpha\left(y, i, a, z_{1} \| z_{2}\right) \text { is maximal } \\
& \quad \text { if } \alpha\left(y, i, a, z_{1} \| z_{2}\right)>\frac{1}{4 n} \\
& \quad \text { output success }
\end{aligned}
$$




\section{fi \\ fi \\ output fail}

The usual Markov style argument shows that the probability that the output is success is at least $\frac{1}{4 n}$ (this is easiest seen by assuming otherwise and computing an upper bound on the expectation of the output in Game 3: it would be less than $\left.\frac{1}{2 n}\right)$.

In Game 5, we choose all of $z$ uniformly at random.

\section{Game 5:}

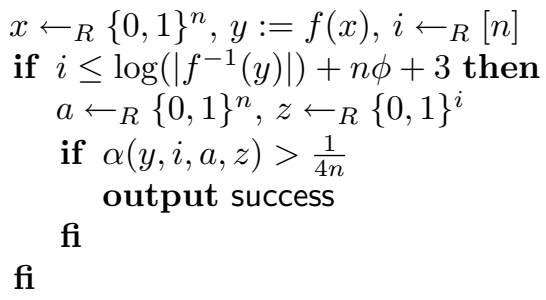

In Game 5, we can assume that $z$ is still chosen as $z_{1} \| z_{2}$. For $z_{1}$, the distribution is the same as in Game 4, for $z_{2}$, we hope that we are lucky and choose it exactly as in Game 4. The length of $z_{2}$ is at most $2 \log (4 n)+n \phi+3$, and thus this happens with probability at least $\frac{1}{128 n^{2}} 2^{-n \phi}$. Thus, in Game 4, with probability at least $\frac{1}{512 n^{3}} 2^{-n \phi}$ the output is success. As mentioned at the start of the proof, in this case running the Goldreich-Levin algorithm with parameter $\frac{1}{4 n}$ will invert $f$ with probability $\frac{1}{2}$, which means that in total we have probability $\Omega\left(\frac{1}{n^{3}}\right) 2^{-n \phi}$ in inverting $f$.

\section{A Pseudorandom Generator from a Pseudo-Entropy Pair}

We now show how we can obtain a pseudorandom generator from a pseudoentropy pair $(g, P)$ as constructed in the last section. The idea here is that we use many (say $k$ ) parallel copies of the function $g$. We can then extract about $k H(g(W))$ bits from the concatenated outputs of $g$, about $k H(W \mid g(W) P(W))$ bits from the concatenated inputs, and about $k(H(P(W) \mid g(W))+\phi)$ bits from the concatenated outputs of $P$. Using the identity $H(g(W))+H(P(W) \mid g(W))+$ $H(W \mid g(W) P(W))=H(W)$, we can see that this will be expanding, and we can say that the $k \phi$ bits of pseudorandomness from $P$ are used to get the expanding property of $h$.

The key lemma in order to prove the security of the construction is the following variant of Impagliazzo's hard-core lemma [9] proven in $[7]^{4}$. For a set $\mathcal{T}$

\footnotetext{
${ }^{4}$ The proposition here is slightly stronger then the corresponding lemma in [7], as we do not require $\gamma$ to be noticeable. It is easy to see that the proof in [7] works in this case as well.
} 
let $\chi_{\mathcal{T}}$ be the characteristic function of $\mathcal{T}$ :

$$
\chi_{\mathcal{T}}(x):= \begin{cases}1 & x \in \mathcal{T} \\ 0 & x \notin \mathcal{T} .\end{cases}
$$

Proposition 3 (Uniform Hard-Core Lemma). Assume that the given functions $g:\{0,1\}^{n} \rightarrow\{0,1\}^{m}, P:\{0,1\}^{n} \rightarrow\{0,1\}, \delta: \mathbb{N} \rightarrow[0,1]$ and $\gamma: \mathbb{N} \rightarrow[0,1]$ are computable in time poly $(n)$, where $\delta$ is noticeable and $\gamma>2^{-n / 3}$.

Further, assume that there exists an oracle algorithm $A^{(\cdot)}$ such that, for infinitely many $n$, the following holds: for any set $\mathcal{T} \subseteq\{0,1\}^{n}$ with $|\mathcal{T}| \geq \delta 2^{n}$, $A^{\chi \mathcal{T}}$ outputs a circuit $C$ satisfying

$$
\mathrm{E}\left[\operatorname{Pr}_{x \leftarrow R} \mathcal{T}[C(g(x))=P(x)]\right] \geq \frac{1+\gamma}{2}
$$

(where the expectation is over the randomness of $A$ ).

Then, there is an algorithm $B$ which calls $A$ as a black box poly $\left(\frac{1}{\gamma}, n\right)$ times, such that

$$
\operatorname{Adv}^{B}(g, P) \geq 1-\delta
$$

for infinitely many $n$. The runtime of $B$ is bounded by poly $\left(\frac{1}{\gamma}, n\right)$ times the runtime of $A$.

The advantage of using Proposition 3 is as follows: in order to get a contradiction, we will use a given algorithm $A$ as oracle to contradict the hardness of a pseudo-entropy pair, i.e., we will give $B$ such that $\operatorname{Adv}^{B}(g, P) \geq 1-$ $H(P(W) \mid g(W))-\phi$. Proposition 3 states that for this it is sufficient to show how to get circuits which perform slightly better than random guessing on a fixed set of size $2^{n}(H(P(W) \mid g(W))+\phi)$, given access to a description of this set. Often, this is a much simpler task.

In the following construction of a pseudorandom generator from a pseudoentropy pair we assume that parameters $\varepsilon$ and $\phi$ are provided (thus they reappear in Theorem 1). The parameter $\varepsilon$ describes how much we lose in the indistinguishability (by making our extractors imperfect), while $\phi$ is the gap of the pseudo-entropy pair.

Further we assume that parameters $\alpha$ and $\beta$ are known which give certain information about the combinatorial structure of the given predicate. We will get rid of this assumption later by trying multiple values for $\alpha$ and $\beta$ such that one of them must be correct. ${ }^{5}$

Lemma 5. Let $g$ and $P$ be efficiently evaluable functions, $g:\{0,1\}^{n} \rightarrow\{0,1\}^{m}$, $P:\{0,1\}^{n} \rightarrow\{0,1\}, \varepsilon:[0,1] \rightarrow \mathbb{N}$, and $\phi:[0,1] \rightarrow \mathbb{N}$ be computable in

${ }^{5}$ Haitner, Harnik, and Reingold [5] construct a pseudo-entropy pair for which $H(P(W) \mid g(W))=\frac{1}{2}$ is fixed. Because of this, they are able to save a factor of $n$ in the seed length under standard assumptions (they do not need to try different values for $\alpha$ ). 
polynomial time, $\phi>\frac{1}{n}$. Assume that parameters $\alpha$ and $\beta$ are such that

$$
\begin{aligned}
& \alpha \leq H(P(W) \mid g(W)) \leq \alpha+\phi / 4 \\
& \beta \leq H(g(W)) \leq \beta+\phi / 4 .
\end{aligned}
$$

There is an efficient to evaluate oracle function $h_{\alpha, \beta, \varepsilon, \phi}^{g}$ with the following properties:

$-h_{\alpha, \beta, \varepsilon, \phi}^{g}$ is expanding,

- $h_{\alpha, \beta, \varepsilon, \phi}^{g}$ has inputs of length $\mathcal{O}\left(n^{3} \frac{1}{\phi^{2}} \log \left(\frac{1}{\varepsilon}\right)\right)$, and

- any algorithm $A$ which distinguishes the output of $h_{\alpha, \beta, \varepsilon, \phi}^{g}$ from a uniform bit string with advantage $\gamma$ can be used to get an oracle algorithm $B^{A}$ satisfying $\operatorname{Adv}^{B}(g, P) \geq 1-H(P(W) \mid g(W))-\phi$ which does poly $\left(\frac{1}{\gamma-\varepsilon}, n\right)$ calls to $A$.

Proof. Let $k:=4096 \cdot\left(\frac{n}{\phi}\right)^{2} \cdot \log \left(\frac{3}{\varepsilon}\right)$ be the number of repetitions (this is chosen such that

$$
\frac{k \phi}{8}=512 \frac{n^{2}}{\phi} \log \left(\frac{3}{\varepsilon}\right)=8 n \sqrt{k \log \left(\frac{3}{\varepsilon}\right)},
$$

which we use later). To simplify notation we set $\lambda:=n-\alpha-\beta-\phi / 2$. Using the notation $w^{k}:=w_{1}\|\ldots\| w_{k}, g^{(k)}\left(w^{k}\right):=g\left(w_{1}\right)\|\ldots\| g\left(w^{k}\right)$ and $P^{(k)}\left(w^{k}\right):=$ $P\left(w_{1}\right)\|\ldots\| P\left(w_{k}\right)$, the function $h_{\alpha, \beta, \varepsilon, \phi}$ is defined as

$$
\begin{aligned}
& h_{\alpha, \beta, \varepsilon, \phi}\left(w^{k}, s_{1}, s_{2}, s_{3}\right):= \\
& \operatorname{Ext}_{k(\beta-\phi / 8)}\left(g^{(k)}\left(w^{k}\right), s_{1}\right)\left\|\operatorname{Ext}_{k(\alpha+7 \phi / 8)}\left(P^{(k)}\left(w^{k}\right), s_{2}\right)\right\| \operatorname{Ext}_{k(\lambda-\phi / 8)}\left(w^{k}, s_{3}\right) .
\end{aligned}
$$

Clearly, the input length is $\mathcal{O}\left(n^{3} \frac{1}{\phi^{2}} \log \left(\frac{1}{\varepsilon}\right)\right)$. We further see by inspection that, excluding the additional randomness $s_{1}, s_{2}$, and $s_{3}$, the function $h$ maps $k n$ bits to at least $k(\alpha+\beta+\lambda)+5 k \frac{\phi}{8}-3=k\left(n-\frac{\phi}{2}\right)+k \frac{5 \phi}{8}-3=k\left(n+\frac{\phi}{8}\right)-3>k n$ bits. Since the additional randomness is also completely contained in the output, $h_{\alpha, \beta, \varepsilon, \phi}$ is expanding for almost all $n$.

We now show that an algorithm $A$ which has advantage $\gamma$ in distinguishing $h_{\alpha, \beta, \varepsilon, \phi}\left(w^{k}, s_{1}, s_{2}, s_{3}\right)$ from a uniform bit string of the same length can be used to predict $P(w)$ given $g(w)$ as claimed above. Per definition the probability that the output is true in the following game is at least $\frac{1+\gamma}{2}$.

\section{Game 0:}

$$
\begin{aligned}
& \left(w_{1}, \ldots, w_{k}\right) \leftarrow_{R}\{0,1\}^{n k} \\
& \begin{array}{l}
b \leftarrow_{R}\{0,1\} \\
\text { if } b=0 \text { then } \\
\quad s_{1} \leftarrow_{R}\{0,1\}^{m k}, v_{1}:=\operatorname{Ext}_{k(\beta-\phi / 8)}\left(g^{(k)}\left(w^{k}\right), s_{1}\right) \\
\quad s_{2} \leftarrow{ }_{R}\{0,1\}^{k}, v_{2}:=\operatorname{Ext}_{k(\alpha+7 \phi / 8)}\left(P^{(k)}\left(w^{k}\right), s_{2}\right)
\end{array} \\
& \quad s_{3} \leftarrow_{R}\{0,1\}^{n k}, v_{3}:=\operatorname{Ext}_{k(\lambda-\phi / 8)}\left(w^{k}, s_{3}\right) \\
& \text { else } \quad \text { (Run A with uniform randomness) } \\
& \qquad v_{1} \leftarrow_{R}\{0,1\}^{m k+k(\beta-\phi / 8)}
\end{aligned}
$$




$$
\begin{aligned}
& v_{2} \leftarrow_{R}\{0,1\}^{k+k(\alpha+7 \phi / 8)} \\
& v_{3} \leftarrow_{R}\{0,1\}^{n k+k(\lambda-\phi / 8)} \\
& \text { fi } \\
& \text { output } b=A\left(v_{1}\left\|v_{2}\right\| v_{3}\right)
\end{aligned}
$$

We now make two transition based on statistical indistinguishability. First, we replace the last part $v_{3}$ in the if-clause of Game 0 with uniform random bits. Because $H(W \mid g(W) P(W))=H(W)-H(g(W))-H(P(W) \mid g(W)) \geq n-$ $\alpha-\beta-\frac{\phi}{2}=\lambda$, Lemma 2 implies that conditioned on the output of $g^{(k)}$ and $P^{(k)}$ (and thus also conditioned on the extracted bits of those outputs) $\operatorname{Ext}_{k \lambda-k \phi / 8}\left(w^{k}, s_{3}\right)=\operatorname{Ext}_{k \lambda-8 n \cdot \sqrt{k \log \left(\frac{3}{\varepsilon}\right)}}\left(w^{k}, s_{3}\right)$ is $\frac{\varepsilon}{3}$-close to the uniform distribution (here we used (6)). Thus this only loses $\varepsilon / 3$ of the advantage $\gamma$ in distinguishing.

Second, we replace $v_{1}$ in the else-clause with $\operatorname{Ext}_{k(\beta-\phi / 8)}\left(g^{(k)}\left(w^{k}\right), s_{1}\right)$. Since $H(g(W)) \geq \beta$, Lemma 2 implies that we only lose $\varepsilon / 3$ in the advantage again. In total, in the following Game 1 we have advantage at least $\gamma-2 \varepsilon / 3$ over random guessing.

\section{Game 1:}

$$
\begin{aligned}
& \left(w_{1}, \ldots, w_{k}\right) \leftarrow_{R}\{0,1\}^{n k} \\
& b \leftarrow_{R}\{0,1\} \\
& \text { if } b=0 \text { then } \\
& \qquad s_{1} \leftarrow_{R}\{0,1\}^{m k}, v_{1}:=\operatorname{Ext}_{k(\beta-\phi / 8)}\left(g^{(k)}\left(w^{k}\right), s_{1}\right) \\
& s_{2} \leftarrow_{R}\{0,1\}^{k}, v_{2}:=\operatorname{Ext}_{k(\alpha+7 \phi / 8)}\left(P^{(k)}\left(w^{k}\right), s_{2}\right) \\
& \quad v_{3} \leftarrow_{R}\{0,1\}^{n k+k(\lambda-\phi / 8)} \\
& \text { else } \\
& \qquad s_{1} \leftarrow_{R}\{0,1\}^{m k}, v_{1}:=\operatorname{Ext}_{k(\beta-\phi / 8)}\left(g^{(k)}\left(w^{k}\right), s_{1}\right) \\
& v_{2} \leftarrow_{R}\{0,1\}^{k+k(\alpha+7 \phi / 8)} \\
& \quad v_{3} \leftarrow_{R}\{0,1\}^{n k+k(\lambda-\phi / 8)} \\
& \text { fi } \quad \\
& \text { output } b=A\left(v_{1}\left\|v_{2}\right\| v_{3}\right)
\end{aligned}
$$

We would like to ignore the parts which are the same in case $b=0$ and $b=1$. It is easy to see that $A^{\prime}$ in Game 2 can be designed such that it calls $A$ with the same distribution as in Game 1.

\section{Game 2:}

$$
\begin{aligned}
& \left(w_{1}, \ldots, w_{k}\right) \leftarrow_{R}\{0,1\}^{n k} \\
& b \leftarrow_{R}\{0,1\} \\
& \text { if } b=0 \text { then } \\
& \quad s \leftarrow_{R}\{0,1\}^{k}, v:=\operatorname{Ext}_{k(\alpha+7 \phi / 8)}\left(P^{(k)}\left(w^{k}\right), s\right) \\
& \text { else } \\
& \quad v \leftarrow_{R}\{0,1\}^{k+k(\alpha+7 \phi / 8)} \\
& \text { fi } \quad \text { output } b=A^{\prime}\left(g^{(k)}\left(w^{k}\right) \| v\right)
\end{aligned}
$$


Later we want to use Proposition 3. Thus we will have an oracle $\chi_{\mathcal{T}}$ which implements the characteristic function of a set $\mathcal{T}$ of size at least $(\alpha+\phi) 2^{n}$. From now on we will use the oracle implicitly in the games by testing whether $w \in \mathcal{T}$.

In Game 3 it is easy to check that in case $b=0$ the distribution with which $A^{\prime}$ is called does not change from Game 2. On the other hand, if $b=1$, then (since $\left.|\mathcal{T}| \geq 2^{n}(\alpha+\phi)\right)$ the $p_{i}$ contain independent random variables with entropy at least $\alpha+\phi$ (where the entropy is conditioned on $g\left(w_{i}\right)$ ). Using Lemma 2 we see that in this case $v$ is $\frac{\varepsilon}{3}$-close to uniform, implying that in Game 3 the advantage of $A^{\prime}$ in predicting $b$ is still $\gamma-\varepsilon$.

\section{Game 3:}

$$
\begin{aligned}
& \left(w_{1}, \ldots, w_{k}\right) \leftarrow_{R}\{0,1\}^{n k} \\
& b \leftarrow_{R}\{0,1\} \\
& \text { for } i \in[n] \text { do } \\
& \quad \text { if } w_{i} \in \mathcal{T} \wedge b=1 \text { then } \\
& \quad p_{i} \leftarrow_{R}\{0,1\} \\
& \quad \text { else } \\
& \quad p_{i}:=P\left(w_{i}\right) \\
& \quad \text { ii } \quad \\
& \begin{array}{l}
\text { od } \quad \leftarrow_{R}\{0,1\}^{k}, v:=\operatorname{Ext}_{k(\alpha+7 \phi / 8)}\left(p^{k}, s\right) \\
\text { output } b=A^{\prime}\left(g^{(k)}\left(w^{(k)}\right) \| v\right)
\end{array}
\end{aligned}
$$

From Game 3, we will now apply a standard hybrid argument to get a predictor for a single position. For this, consider Game 4.

\section{Game 4:}

$$
\begin{aligned}
& \left(w_{1}, \ldots, w_{k}\right) \leftarrow_{R}\{0,1\}^{n k} \\
& j \leftarrow_{R}[n] \\
& \text { for } i \in\{1, \ldots, j-1\} \text { do } \\
& \quad \text { if } w_{i} \in \mathcal{T} \text { then } p_{i} \leftarrow_{R}\{0,1\} \text { else } p_{i}:=P\left(w_{i}\right) \mathbf{f i} \\
& \text { od } \\
& \text { for } i \in\{j+1, \ldots, n\} \text { do } p_{i}:=P\left(w_{i}\right) \text { od } \\
& b \leftarrow_{R}\{0,1\} \\
& \text { if } w_{j} \in \mathcal{T} \wedge b=1 \text { then } p_{j} \leftarrow_{R}\{0,1\} \text { else } p_{j}:=P\left(w_{j}\right) \mathbf{f i} \\
& s \leftarrow R\{0,1\}^{k}, v:=\operatorname{Ext}_{k(\alpha+7 \phi / 8)}\left(p^{k}, s\right) \\
& \text { output } b=A^{\prime}\left(g^{(k)}\left(w^{(k)}\right) \| v\right)
\end{aligned}
$$

The distribution $A^{\prime}$ is called in Game 4 in case $b=0$ and $j=1$ is the same as in Game 3 in case $b=0$; the distribution used in Game 4 in case $b=1$ and $j=n$ is the same as in Game 3, in case $b=1$. Further, the distribution in Game 4 does not change if $b$ is set from 1 to 0 and $j$ is increased by one. This implies that the advantage of $A^{\prime}$ in predicting $b$ is $(\gamma-\varepsilon) / k$.

In Game 5, we replace $A^{\prime}$ with $A^{\prime \prime}$ which does all the operations common in case $b=0$ and $b=1$ (the $w$ chosen in Game 5 corresponds to $w_{j}$ in Game 4 , and $A^{\prime \prime}$ chooses the value of $j$, and all other $w_{i}$ before calling $A^{\prime}$ ). 


\section{Game 5:}

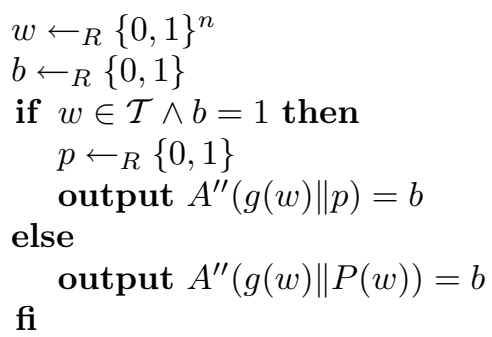

An easy calculation now yields that for $w \leftarrow_{R} \mathcal{T}$ and $p \leftarrow_{R}\{0,1\}$ the probabillity that

$$
1 \oplus p \oplus A^{\prime \prime}(g(w) \| p)=P(w)
$$

is at least $\frac{1}{2}+\frac{\gamma-\varepsilon}{k}$. Since this works for any $\mathcal{T}$ with $|\mathcal{T}| \geq(\alpha+\phi) 2^{n}$, and thus for every $\mathcal{T}$ with $|\mathcal{T}| \geq(H(P(W) \mid g(W))+\phi) 2^{n}$, we can apply Proposition 3 and get the lemma.

With this lemma, we can now prove Theorem 1.

Proof (of Theorem 1). Given $\varepsilon$ and $\phi$, we use the construction of Lemma 4 to get a predicate which we use in the construction of Lemma 5 for $\frac{16 n}{\phi^{2}}$ different values of $\alpha$ and $\beta$ (note that $0 \leq H(g(W)) \leq n$ ), such that for at least one of those choices the requirements of Lemma 5 hold. Further, in those applications we use $\varepsilon^{\prime}:=\Omega\left(\varepsilon \frac{\phi^{4}}{n^{5}}\right)$ in place of $\varepsilon$. Since $\varepsilon^{\prime}=\Omega\left(\varepsilon^{10}\right)$, this satisfies $\mathcal{O}\left(\log \left(\frac{1}{\varepsilon}\right)\right)=\mathcal{O}\left(\log \left(\frac{1}{\varepsilon^{\prime}}\right)\right)$.

For every choice of $\alpha$ and $\beta$ we concatenate $h_{\alpha, \beta, \varepsilon^{\prime}, \phi}:\{0,1\}^{\ell} \rightarrow\{0,1\}^{\ell+1}$ with itself, in order to obtain a function $h_{\alpha, \beta, \varepsilon^{\prime}, \phi}^{\prime}:\{0,1\}^{\ell} \rightarrow\{0,1\}^{16 n \phi^{-2} \ell+1}$, i.e., the first part of the output of $h_{\alpha, \beta, \varepsilon^{\prime}, \phi}$ is used to call $h_{\alpha, \beta, \varepsilon^{\prime}, \phi}$ again, and this process is repeated $16 n \phi^{-2} \ell \in \mathcal{O}\left(n^{5} \frac{1}{\phi^{4}}\right)$ times, and every time we get one more bit of the final output.

The function $h_{\varepsilon, \phi}:\{0,1\}^{16 n \phi^{-2} \ell} \rightarrow\{0,1\}^{16 n \phi^{-2} \ell+1}$ divides its input into $\frac{16 n}{\phi^{2}}$ blocks of length $\ell$, calls the functions $h_{\alpha, \beta, \varepsilon^{\prime}, \phi}^{\prime}$ with seperate blocks, and XORs the outputs.

Assume now that an algorithm $A$ can distinguish the output of $h_{\varepsilon, \phi}$ from a unifrom random string with advantage $\gamma$. For every choice of $\alpha$ and $\beta$ (and in particular the choice which satisfies the requirements of Lemma 5) we try the following to invert $f$. First, since we can simulate the other instances, we see that we have advantage $\gamma$ in distinguishing the output of $h_{\alpha, \beta, \varepsilon^{\prime}, \phi}^{\prime}$ from a random string. We can use the hybrid argument to get an algorithm which has advantage $\gamma^{\prime}:=\Omega\left(\gamma \phi^{4} n^{-5}\right)$ in distinguishing the output of $h_{\alpha, \beta, \varepsilon^{\prime}, \phi}$ from a random string. From Lemma 5 we get an algorithm which predicts $P$ from $g$ with advantage at least $1-H(P(W) \mid g(W))-\phi$, and the number of calls is bounded by $\operatorname{poly}\left(\frac{1}{\gamma^{\prime}-\varepsilon^{\prime}}, n\right)=\operatorname{poly}\left(\frac{1}{\gamma-\varepsilon}, n\right)$. Finally, Lemma 4 implies that we can get an inverter with the claimed complexity and success probability. 


\section{Acknowledgments}

I would like to thank Ueli Maurer, Krzysztof Pietrzak, Dominik Raub, Renato Renner, Johan Sjödin, and Stefano Tessaro for helpful comments and discussions. Also, I would like to thank the anonymous referees who provided helpful criticism about the presentation of this paper. I was supported by the Swiss National Science Foundation, project no. 200020-103847/1.

\section{References}

1. Manuel Blum and Silvio Micali. How to generate cryptographically strong sequences of pseudo-random bits. Siam Journal on Computation, 13(4):850-864, 1984.

2. Oded Goldreich, Shafi Goldwasser, and Silvio Micali. How to construct random functions. Journal of the ACM, 33(4):792-807, 1986.

3. Oded Goldreich, Hugo Krawczyk, and Michael Luby. On the existence of pseudorandom generators. Siam Journal on Computation, 22(6):1163-1175, 1993.

4. Oded Goldreich and Leonid A. Levin. A hard-core predicate for all one-way functions. In Proceedings of the Twenty First Annual ACM Symposium on Theory of Computing, pages 25-32, 1989.

5. Iftach Haitner, Danny Harnik, and Omer Reingold. On the power of the randomized iterate. Technical Report TR05-135, Electronic Colloquium on Computational Complexity (ECCC), 2005.

6. Johan Håstad, Russell Impagliazzo, Leonid A. Levin, and Michael Luby. A pseudorandom generator from any one-way function. Siam Journal on Computation, 28(4):1364-1396, 1999.

7. Thomas Holenstein. Key agreement from weak bit agreement. In Proceedings of the Thirty Seventh Annual ACM Symposium on Theory of Computing, pages 664-673, 2005.

8. Thomas Holenstein and Renato Renner. On the smooth Rényi entropy of independently repeated random experiments. In preparation, 2005.

9. Russell Impagliazzo. Hard-core distributions for somewhat hard problems. In The 36th Annual Symposium on Foundations of Computer Science, pages 538-545, 1995.

10. Russell Impagliazzo, Leonid A. Levin, and Michael Luby. Pseudo-random generation from one-way functions (extended abstract). In Proceedings of the Twenty First Annual ACM Symposium on Theory of Computing, pages 12-24, 1989.

11. Michael Luby and Charles Rackoff. How to construct pseudorandom permutations from pseudorandom functions. Siam Journal on Computation, 17(2):373-386, 1988.

12. Michael Luby and Avi Wigderson. Pairwise independence and derandomization. Technical Report ICSI TR-95-035, International Computer Science Institute, Berkeley, CA, 1995.

13. Moni Naor. Bit commitment using pseudorandomness. Journal of Cryptology, 4(2):151-158, 1991.

14. Victor Shoup. Sequences of games: a tool for taming complexity in security proofs. Technical Report 332, http://eprint.iacr.org/2004/332, 2004. 
15. Hoeteck Wee. On obfuscating point functions. In Proceedings of the Thirty Seventh Annual ACM Symposium on Theory of Computing, pages 523-532, 2005.

16. Andrew C. Yao. Theory and applications of trapdoor functions (extended abstract). In The 23rd Annual Symposium on Foundations of Computer Science, pages $80-91,1982$. 